\title{
Anticancer activity of sulforaphane against human hepatoblastoma cells involves apoptosis, autophagy and inhibition of $\beta$-catenin signaling pathway
}

\author{
Junshan Lin ${ }^{1}$, Yali Xü ${ }^{1}$ Xing Zhao ${ }^{2}$, Zhixin Qiü
}

${ }^{1}$ Department of Pediatric Surgery, The First Affiliated Hospital of Fujian Medical University, Fuzhou, Fujian Province, China

${ }^{2}$ First Clinical Medical College, The First Affiliated Hospital of Fujian Medical

University, Fuzhou, Fujian Province, China

Submitted: 19 February 2020

Accepted: 22 March 2020

Arch Med Sci

DOI: https://doi.org/10.5114/aoms.2020.96077

Copyright $\odot 2020$ Termedia \& Banach

\section{Abstract}

Introduction: Sulforaphane is an active isothiocyanate and has been reported to exhibit many pharmacological and biological activities including anticancer activity. The current study was undertaken to examine its anticancer activity against human hepatoblastoma.

Material and methods: MTT and clonogenic assays were used to assess the antiproliferative effects of sulforaphane. Annexin V/PI staining and immunofluorescence microscopy were used to monitor the induction of apoptosis. GFP-LC3 transfection and fluorescence microscopy were used to investigate the induction of autophagy. Cell migration and invasion were assessed through wound healing and transwell chamber assay. Protein expression was determined by western blot analysis,

Results: The results indicated that sulforaphane induced antiproliferative effects on the hepatoblastoma cell lines and exhibited an $I C_{50}$ of $10 \mu \mathrm{M}$ against the Hepu1 and HepU2 cells as compared to the $\mathrm{IC}_{50}$ of $90 \mu \mathrm{M}$ against the normal THLE-2 cells. The antiproliferative effects were observed to be mediated via apoptosis which was accompanied by upregulation of Bax and suppression of $\mathrm{Bcl}-2$ together with activation of caspase- 3 and PARP cleavage. Sulforaphane also induced autophagy and the $\beta$-catenin signaling pathway. In addition, cell migration and cell invasion of the hepatoblastoma cells were also suppressed upon sulforaphane treatment.

Conclusions: The results indicated that sulforaphane is a potential anticancer agent and may be considered as a lead molecule in the development of hepatoblastoma chemotherapy.

Key words: isothiocyanate, sulforaphane, hepatoblastoma, apoptosis, autophagy.

\section{Introduction}

Hepatoblastoma (HB) is a destructive liver malignancy, found mostly in children [1-3]. The incidence of HB has increased rapidly in the past few years with higher incidence rates among children aged 0-4 years. Children aged > 15 years are rarely found with $\mathrm{HB}$ [4]. The risk factors associated with the development of HB have not been fully understood. Tobacco consumption, low body weight and a few inherited syndromes including the Beckwith-Wiedemann syndrome may be considered as

\author{
Corresponding author: \\ Yali Xu \\ Department \\ of Pediatric Surgery \\ The First Affiliated \\ Hospital of Fujian \\ Medical University \\ 20 Chazhong Road \\ Fuzhou 350004 \\ Fujian Province, China \\ Phone/fax: +865918798 \\ 3333 \\ E-mail: \\ fyyy83357199@126.com
}


major risk factors contributing to $\mathrm{HB}$ development [5]. Complete surgical resection of the tumor remains the cornerstone in $\mathrm{HB}$ management and it is associated with higher efficacy and increased survival chances. However, extensive uni/multi focal primary tumor, disease relapse, distant metastasis and lost surgery chances contribute to a poor 5-year survival rate $(20-30 \%)[6,7]$. The last four decades have seen potential advances in the understanding and treatment of cancer. In one such attempt, doxorubicin (DO) and cisplatin (PLA) were used synergistically to manage $\mathrm{HB}$, which led to enhanced prognosis. Thus, lack of effective treatment strategies and anti-HB chemopreventives have started a hunt for novel anti-HB therapeutic sulforaphanes. Isothiocyanates are a naturally occurring set of glucosinolates prevailing among cruciferous vegetables including cabbage and broccoli. Sulforaphane, an active isothiocyanate, shows diverse biological and medicinal properties [8]. Sulforaphane has been reported to have anticancer properties as well as acting as a prime inducer of phase II enzymes [9-11]. Sulforaphene, an analog of sulforaphane, has also been reported to suppress carcinogenesis via the MAP kinase pathway [12]. Though sulforaphane bears significant anticancer potential with low toxicity, high isolation cost limits its application clinically. Sulforaphane was observed to induce anticancer effects against epithelial Hep-2 carcinoma cells and Barrett's adenocarcinoma [13, 14]. Sulforaphane has been reported to exhibit anticancer activity due its apoptosis- as well as autophagy-inducing potential [15-17]. In addition, sulforaphane has shown synergistic effects with anticancer activity of taxanes, thereby eliminating the stem cells in triple negative breast carcinoma. Thus, taking into consideration the reported anticancer activity of sulforaphane, the current study was designed to investigate its anticancer behavior against HB. In addition, sulforaphane was testified for apoptosis induction, autophagy induction and inhibition of the $\beta$-catenin signaling pathway.

\section{Material and methods}

\section{Determination of cellular viability}

MTT assay was performed to examine the cellular viability of HepU1, HepU2 and normal THLE-2 cells after sulforaphane treatment. Each cell line was placed on 96-well flat bottom microtiter cultural plates with a concentration of $4 \times 10^{4}$ cells/ well and precultured for a period of $24 \mathrm{~h}$ with incubation. Afterwards, all three cell lines were subjected to sulforaphane treatment $(2.5,5,10,20$, $40,80,160,320$ and $640 \mu \mathrm{M})$ in $\mathrm{CO}_{2}(5 \%)$ incubator for $24 \mathrm{~h}$ and at $37^{\circ} \mathrm{C}$. Each well of the culture plate was then supplied with $0.2 \mathrm{mg} / \mathrm{ml}$ of fresh
MTT solution. After $4 \mathrm{~h}$ of additional incubation at $37^{\circ} \mathrm{C}$, detergent solution DMSO was used for dissolving formazan crystals. Finally, an Asys UVM 340 Microplate reader, Biochrom, was used to take absorbance at $570 \mathrm{~nm}$ for the measurement of optical density (OD).

\section{Clonogenic assay}

For clonogenic assessment of HepU1 and HepU2 cell lines after sulforaphane exposure a clonogenic assay was implemented. Briefly, both the cancerous cell lines were loaded onto 6-well plates with 300-400 cells/well. Afterwards, each well was supplemented with varying sulforaphane doses of control, 5, 10 and $20 \mu \mathrm{M}$ for 10 days. After 10 days of exposure when cell colonies became visible, complete medium was removed and cell colonies were washed three times using PBS (phosphate buffer saline). Both types of cell lines were stained for 15 min with crystal violet after fixation with paraformaldehyde (4\%). Clusters of $\geq 50$ cells were considered as cell colonies and were photographed with a camera.

\section{Annexin V/PI staining}

Quantification of apoptosis in HepU1 and HepU2 cells was accomplished by performing annexin V/PI staining assay. For that the FITC Annexin V Apoptosis Detection Kit I (BD Biosciences) was used in accordance with provided guidelines. In brief, sub-culture of both cell types at $2.5 \times 10^{4}$ concentration was performed in 6-well plates. Afterwards, different sulforaphane doses, viz control, 5, 10 and $20 \mu \mathrm{M}$, were added each plate for a period of $24 \mathrm{~h}$. Thereafter, both treated cell types were harvested and subjected to PBS washing twice followed by resuspension in $1 \times$ binding buffer. Aliquots of $10^{4}$ cells were subjected to FITC Annexin V and PI (5 $\mu$ l each) staining. Finally, a flow cytometer (BD Biosciences, United States) was used to examine each sample.

\section{Immunofluorescence for cleaved caspase-3 analysis}

The sulforaphane $(0,510$ and $20 \mu \mathrm{M})$ treated HepU1 and HepU2 cells were cultured and subjected to fixation on coverslips with paraformaldehyde for $20 \mathrm{~min}$ at $25^{\circ} \mathrm{C}$. This was followed by washing with PBS incubation for 30 min with $1 \%$ bovine serum albumin (BSA) to prevent the blocking of antibodies non-specifically. The cells were then subjected to incubation with primary antibody (1 : 200 dilution) and washing with PBS. Next the cell samples were subjected to incubation with Alexa Fluor 488-conjugated goat antirabbit IgG secondary antibody (1 : 200) in 1\% BSA for $1 \mathrm{~h}$ in the dark at $25^{\circ} \mathrm{C}$. The samples were then 
subjected to counterstaining with DAPI for $7 \mathrm{~min}$. Finally, the coverslips were subjected to visualization using a fluorescence microscope (200x magnification).

\section{Autophagy detection}

The GFP-LC3 vectors were transfected into the HepU1 and HepU2 cells and then treated with different doses of sulforaphane $(0,5,10$ and $20 \mu \mathrm{M})$ and then examined by fluorescence microscopy.

\section{Wound healing migration assay}

Wound healing migration assay was performed to monitor the rate of migration in HepU1 and HepU2 cells after sulforaphane exposure (control, 5,10 and $20 \mu \mathrm{M}$ ). Both the cell lines were cultured in 6-well plates until they reached $80 \%$ of growth confluence followed by PBS washing once. Thereafter, a sterile pipette tip was used to scratch a wound in both types of treated cell lines including controls and pictured. All well plates were then incubated at $37^{\circ} \mathrm{C}$ for $24 \mathrm{~h}$ followed by taking further images with an inverted microscope.

\section{Transwell chambers invasion assay}

Transwell chambers were coated with Matrigel in order to assess cell invasion in HepU1 and HepU2 cells after test sulforaphane treatment. Briefly, both the cell lines were transfected for $24 \mathrm{~h}$ with sulforaphane with various concentrations, viz control, 5, 10 and $20 \mu \mathrm{M}$. Afterwards, $200 \mathrm{ml}$ of transfected cells were plated onto upper transwell chambers containing medium and $10 \%$ FBS (fetal bovine serum). The bottom chambers were only plated with medium. After $2 \mathrm{~h}$ of incubation, non-invaded cells were removed, and invaded cells were fixed using methyl alcohol followed by staining with crystal violet (CV). Finally, to estimate the number of invaded cells, an inverted microscope with 200x magnification was used.

\section{Western blotting assay}

After exposure with sulforaphane, HepU1 and HepU2 cell lines were lysed using RIPA buffer (icecold). Normalization of the lysates was accomplished by the Enhanced BCA Protein Assay Kit (Beyotime). 10-15\% SDS-PAGE was used to separate proteins and proteins were electrophoretically transferred to PVDF membranes. Then blocking of membranes was performed using non-fat milk (5\%) followed by blotting of membranes with primary antibodies overnight at $4^{\circ} \mathrm{C}$ (LC3B-I, II, Beclin-1, Atg7, Bax, Bcl-2, caspase-3, PARP, $\beta$-catenin and cyclin D1). Species-specific peroxidase-conjugated secondary antibodies were used to detect proteins after incubation. A chemiluminescence
Kit (Millipore, Plano, TX, USA) was used to detect immunoreactive bands and visualization was done with a Vilber Fusion FX6-XT imaging system.

\section{Statistical analysis}

All the individual experiments were performed three times and the results were stated as mean \pm SD (standard deviation). Student's $t$ test and oneway ANOVA followed by Tukey's test were used for all statistical calculations taking $p<0.05$ as statistically significant.

\section{Results}

\section{Inhibition of HB growth by sulforaphane}

Cell growth rate in sulforaphane treated $\mathrm{HB}$ cells (HepU1 and HepU2) and treated normal liver cells (THLE2) was monitored through MTT assay. The results demonstrated that the proliferation rate in sulforaphane-treated cancer cell lines (HepU1 and HepU2) was suppressed potentially and dose reliantly. The viability in the case of HepU1 cells decreased from $100 \%$ to almost $5 \%$ at a sulforaphane concentration varying from 0-640 $\mu \mathrm{M}$ (Figure $1 \mathrm{~A}$ ). In the case of sulforaphane treated HepU2 cells, viability was observed to decrease remarkably from $100 \%$ to almost $2 \%$ (Figure 1 B). Against the normal THLE-2 liver cell line, sulforaphane-induced cytotoxicity was comparatively lower (Figure $1 \mathrm{C}$ ). Therefore, MTT assay results revealed the antiproliferative nature of sulforaphane against HB. Clonogenic assay indicated that after 10 days of sulforaphane treatment the numbers of cancer cell colonies (both HepU1 and HepU2 cell colonies) were significantly decreased. The number of colonies decreased to almost to $20 \%$ in HepU1 (Figure 1D) and almost to $8 \%$ on sulforaphane treatment (Figure $1 \mathrm{E}$ ).

\section{Sulforaphane induced apoptosis in HB cell lines}

Sulforaphane induced antiproliferative effects on two different HB cell lines, HepU1 and HepU2; therefore, an attempt was made to reveal its underlying mechanism. Annexin V/PI staining assay was performed to check different quantities of apoptotic HepU1 and HepU2 cells after sulforaphane treatment. In both the cell lines, sulforaphane triggered the induction of apoptotic cell death. The apoptosis percentage was $1.5 \%, 6.7 \%$, $12.7 \%$ and $19.11 \%$ in the case of HepU1 and $2.4 \%$, $12.1 \%, 17.7 \%$ and $24.9 \%$ at $0,5,10$ and $20 \mu \mathrm{M}$ dosage of sulforaphane (Figure 2). Therefore, it was evidenced that anti-cell growth effects of sulforaphane are apoptosis mediated. Further, a DAPI staining assay was performed to monitor nuclear morphology in the case of both the cell lines. 
A

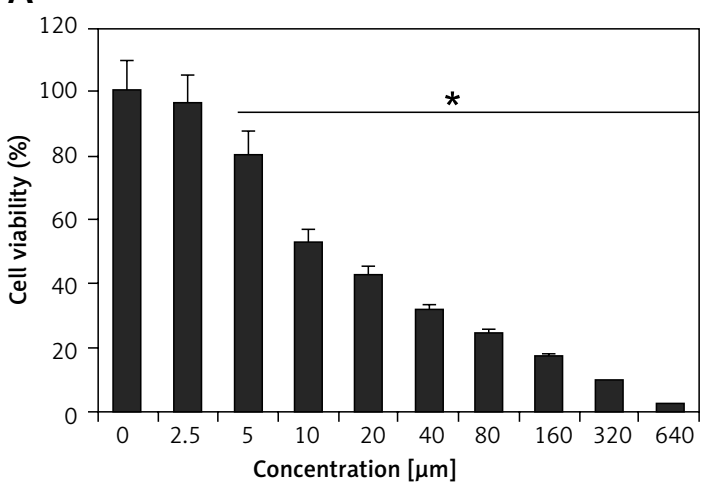

C

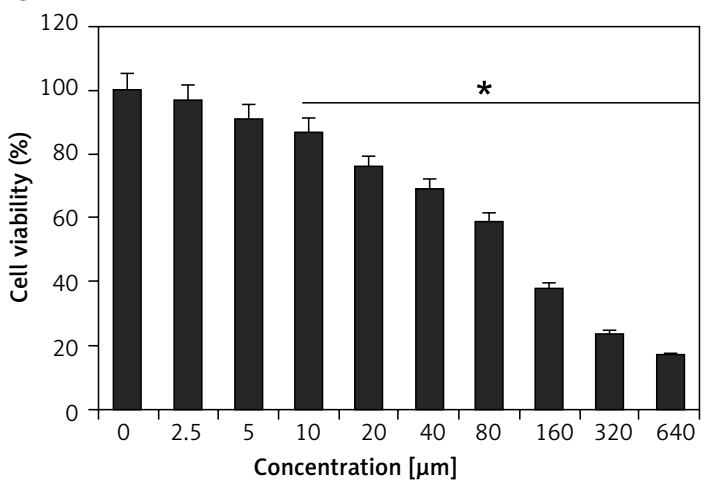

D

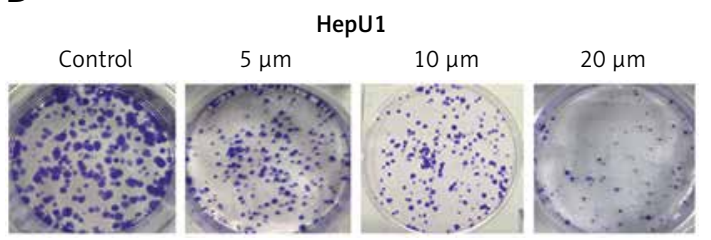

B

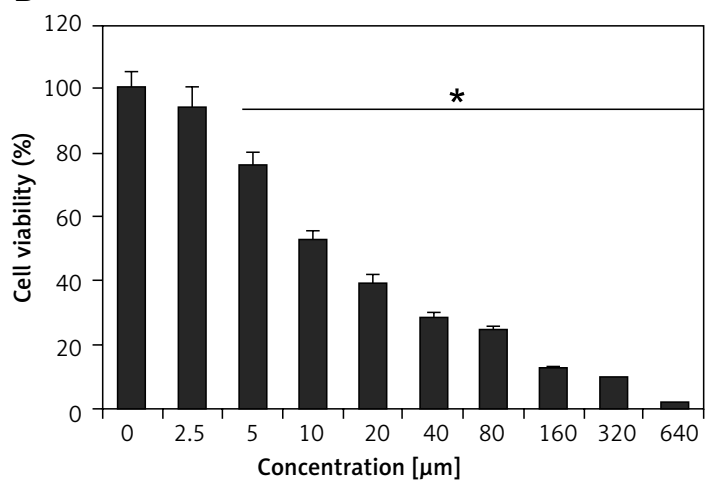

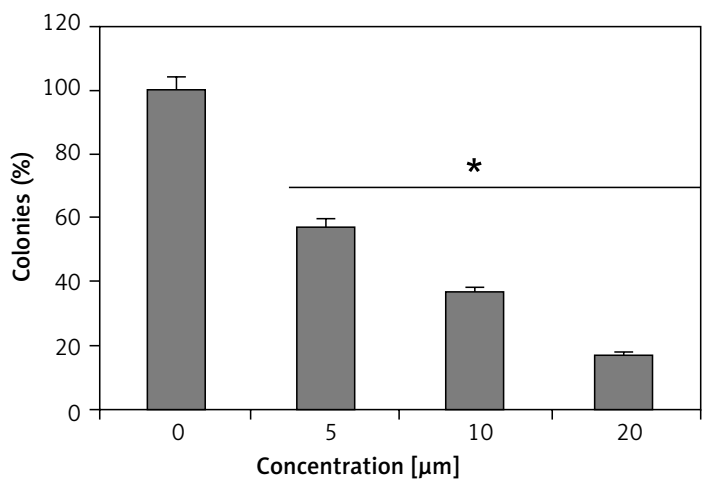
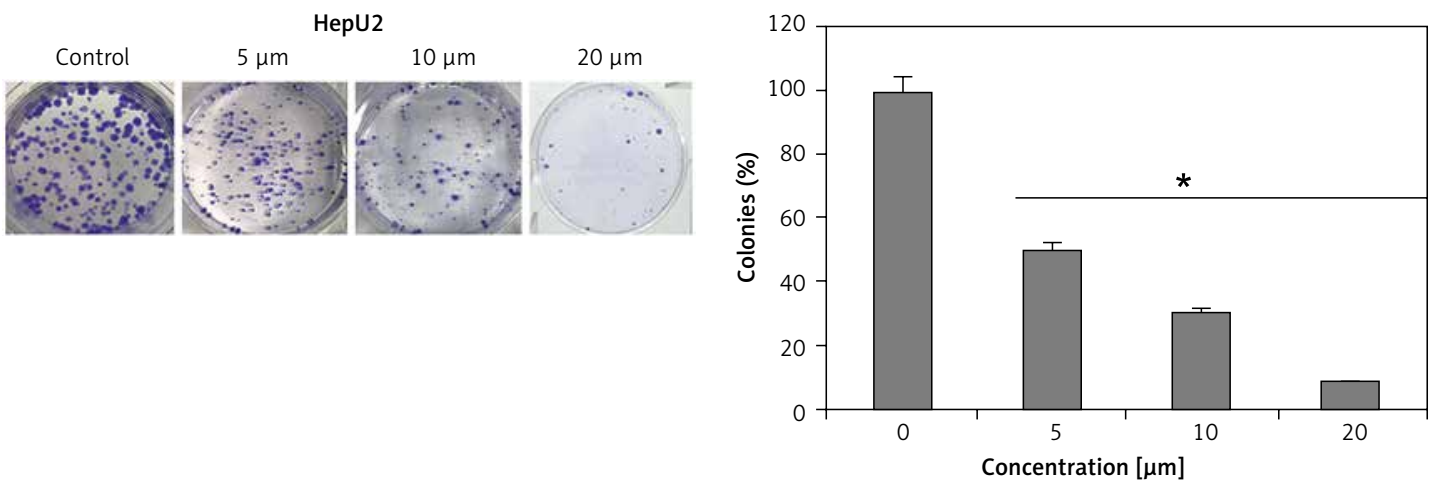

Figure 1. A - Viability of HepU1 cells after treatment with indicated sulforaphane doses. B - Viability of HEepU2 cells after treatment with indicated sulforaphane doses. C - Viability of THLE-2 cells after treatment with indicated sulforaphane doses. D - Representation of number of HepU1 cell colonies after indicated HEPU1 sulforaphane doses. $\mathbf{E}$ - Representation of number of HepU2 cell colonies after indicated HepU1 sulforaphane doses. All the individual experiments were performed three times and the results were stated as mean \pm SD (standard deviation) $\left({ }^{*} p<0.05\right)$ 
The results indicated nuclear fragmentation and enhanced fluorescence indicated higher amounts of cleaved caspase-3 (Figure 3 A) suggestive of apoptotic cell death in HepU1 and HepU2 cell lines after sulforaphane exposure. To further verify the apoptosis-inducing tendency of sulforaphane,

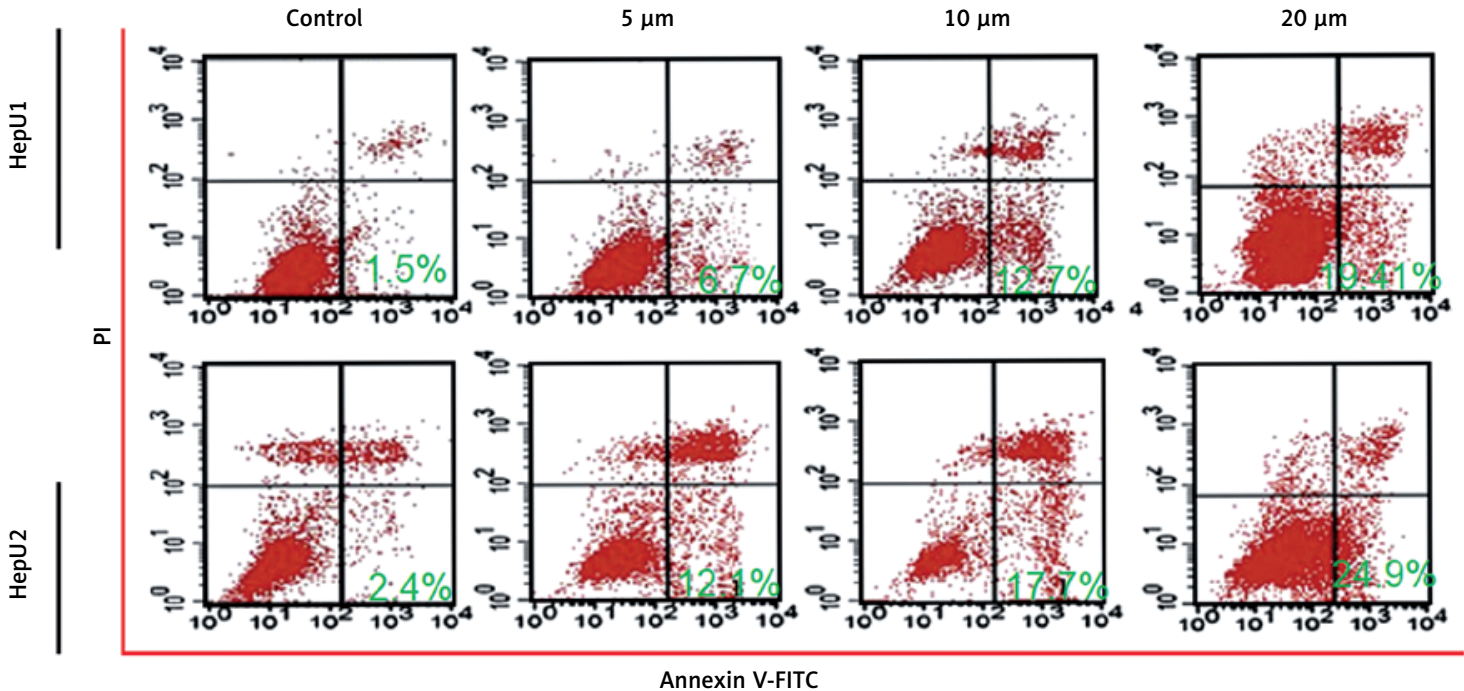

Figure 2. Annexin V/PI staining assay results through flow cytometry. Results presenting early, late apoptosis and necrotic HepU1 and HepU2 cell populations at indicated sulforaphane doses. All the individual experiments were performed three times
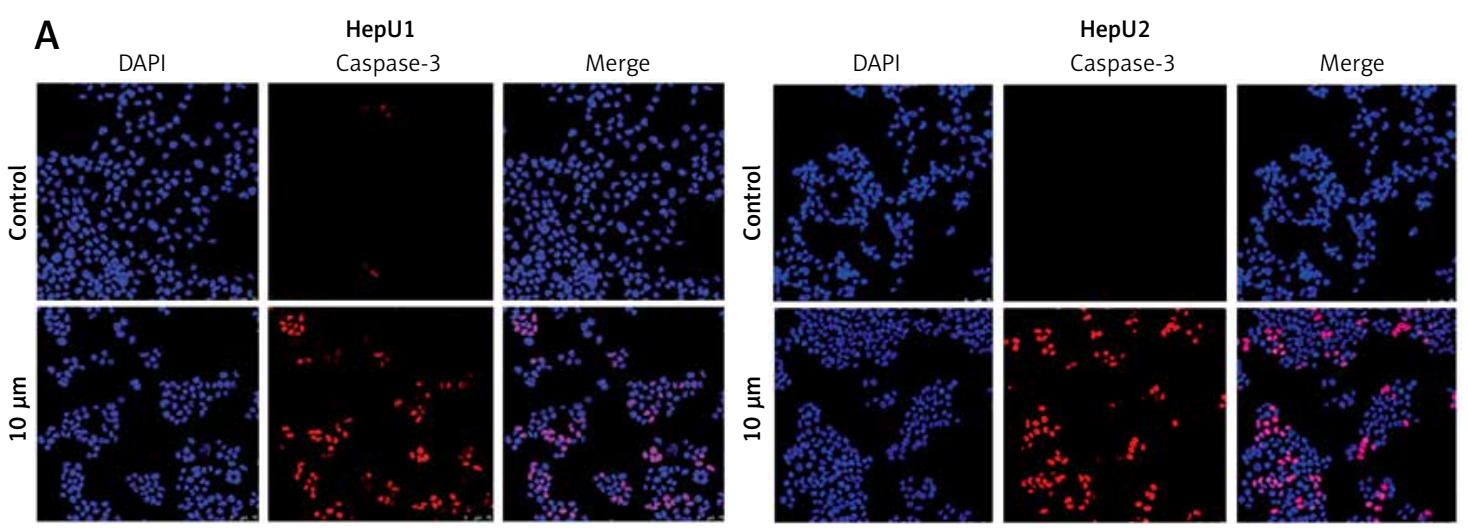

B

HepU1 Sulforaphane $[\mu \mathrm{m}]$

$\mathrm{HepU} 2$ Sulforaphane $[\mu \mathrm{m}]$
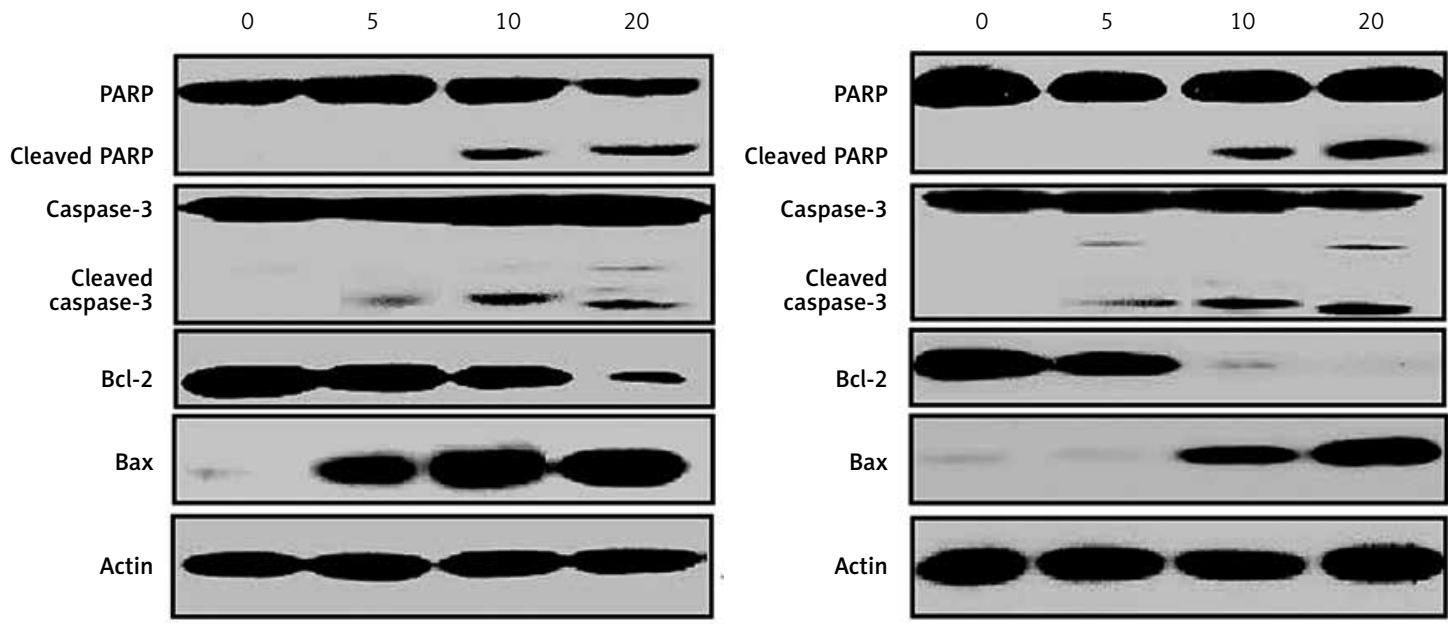

Figure 3. A - Images representing the results of immunofluorescent imaging showing the induction of cleaved caspase-3 in HepU1 and HepU2 cells after sulforaphane treatment. B - Western blotting assay representing activity of pro-apoptotic and anti-apoptotic proteins. All experiments were performed three times 
western blotting assay was performed. The results revealed that the levels of PARP and caspase- 3 remained unchanged in both treated cell lines but cleaved PARP and cleaved caspase- 3 showed enhanced expression. It was also observed that the expression of Bcl-2 was suppressed significantly and that of Bax enhanced tremendously on sulforaphane application (Figure $3 \mathrm{~B}$ ).

Sulforaphane induced autophagy in HB cells

Sulforaphane was evaluated for its autophagy-inducing potency as well. Enhanced activity of LC3 proteins and autophagosome formation clearly hallmark the autophagic cell death. Fluorescence microscopy indicated increased LC3 proteins which indicated autophagic cell death in both HepU1 and HepU2 cell lines (Figure 4 A). Further, western blotting assay was performed to monitor autophagy-associated protein expression. The results in both cell lines indicated that the activity of Atg-7, Beclin-1, LC3-I and LC3-II was enhanced with increased sulforaphane concentrations. Thus, these results indicate the autophagy-inducing potency of sulforaphane.

A

Control

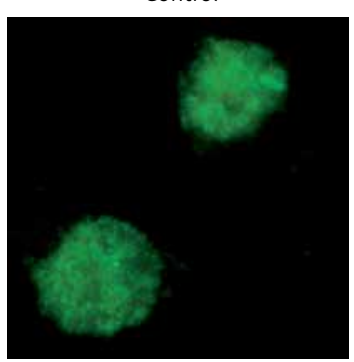

Control

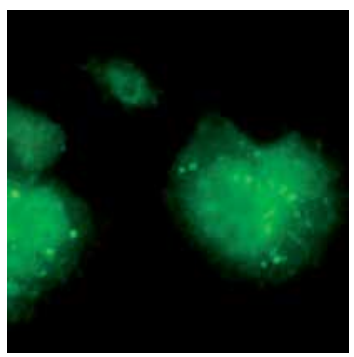

B

HepU1

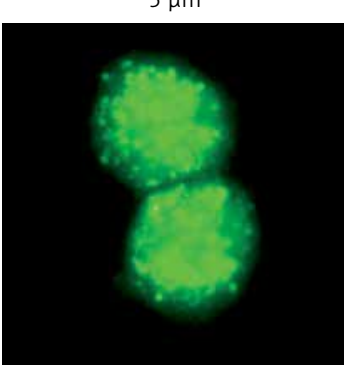

$10 \mu \mathrm{m}$

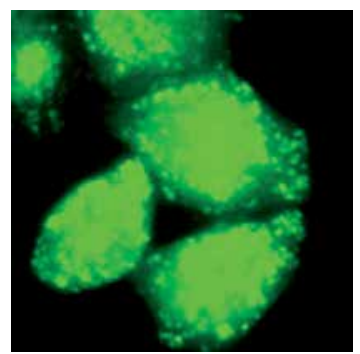

$20 \mu \mathrm{m}$

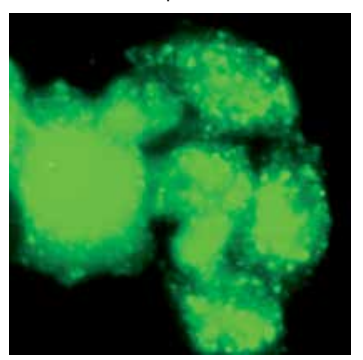

HepU2

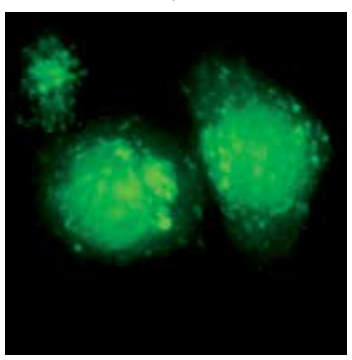

$10 \mu \mathrm{m}$
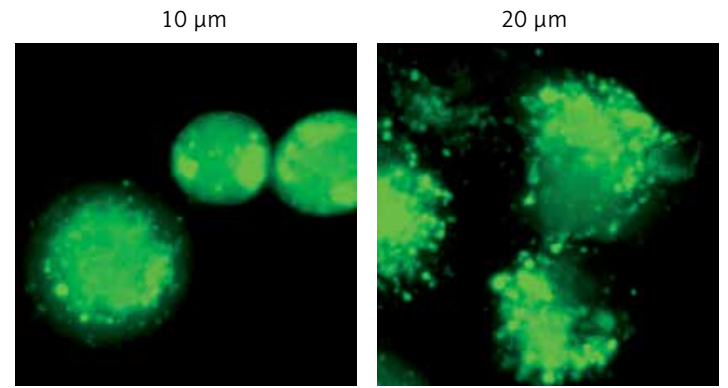

lforaphane $[\mu \mathrm{m}]$

$10 \quad 20$
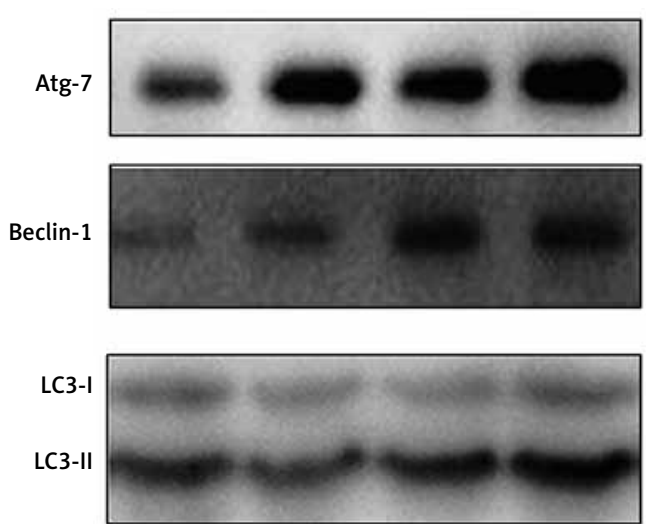

Actin

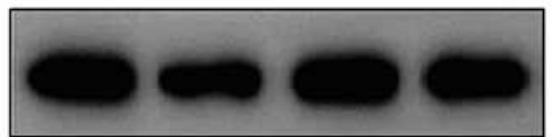

HepU2

Sulforaphane $[\mu \mathrm{m}]$
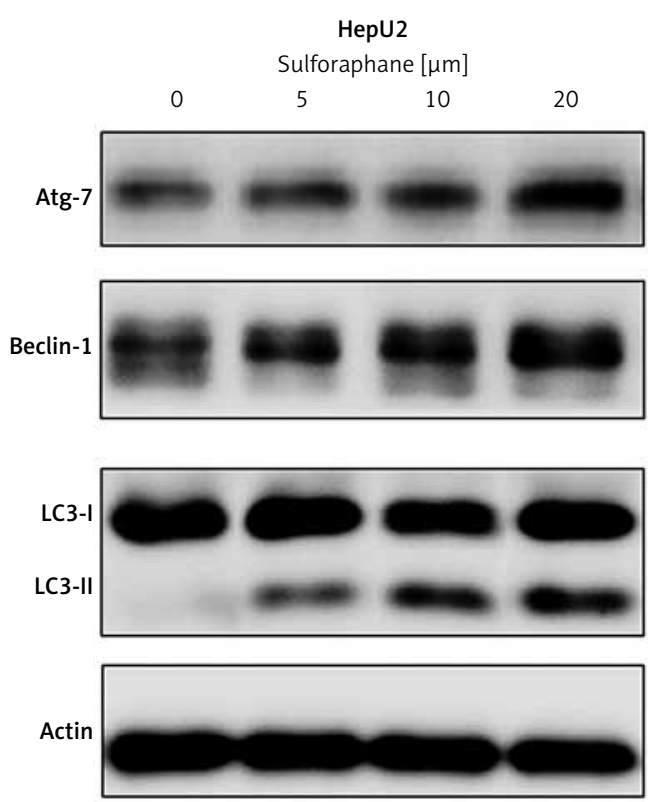

Figure 4. A - Effect of sulforaphane on expression of LC3 in HepU1 and HepU2 cells transfected with GFP-LC3. B - Western blotting assay presenting activity of pro-autophagy proteins Atg-7, Beclin-1, LC3-I and LC3-II. Experiments were repeated three times 


\section{Sulforaphane inhibits cell migration and} invasion of HB cells

Cell migration was monitored through wound width determination after sulforaphane exposure. The results from the wound healing assay revealed reduced migratory potency of HepU1 and HepU2 cells upon sulforaphane treatment (Figure $5 \mathrm{~A}$ ). The width of the wound in the case of controls began to almost close after $24 \mathrm{~h}$ and a fresh scratched wound was observed in the case of treated cell lines. Cell invasion in both types of cell lines was monitored in transwell chambers coated with Matrigel. The percentage of invaded cells in controls was taken as $100 \%$ and the rate of invasion was observed to be declining with increased doses of sulforaphane (Figure 5 B).

\section{Sulforaphane inhibits $\beta$-catenin signaling pathway}

As the $\beta$-catenin signaling pathway is an important pathway in cellular growth and differentiation, targeting it in a cancer cell results in cell
A

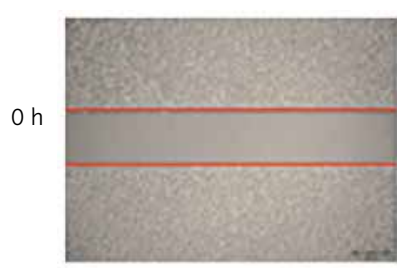

$24 \mathrm{~h}$

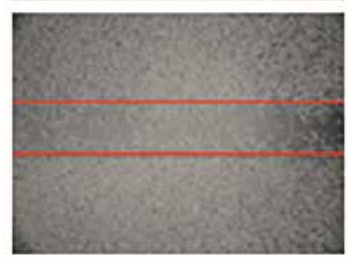

HepU1
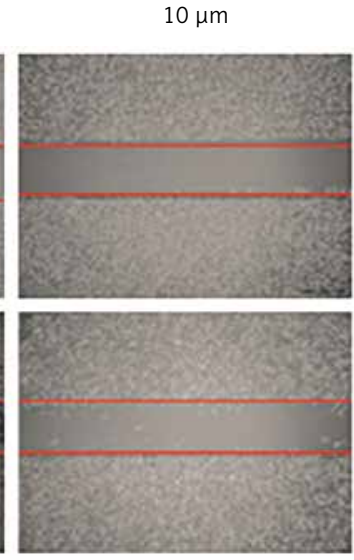

HepU1

Control

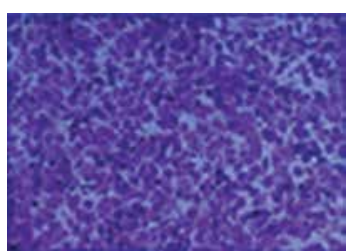

$10 \mu \mathrm{m}$

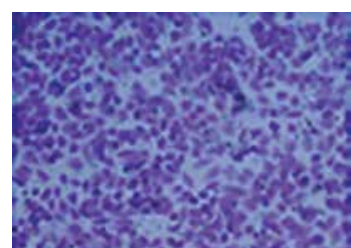

HepU2
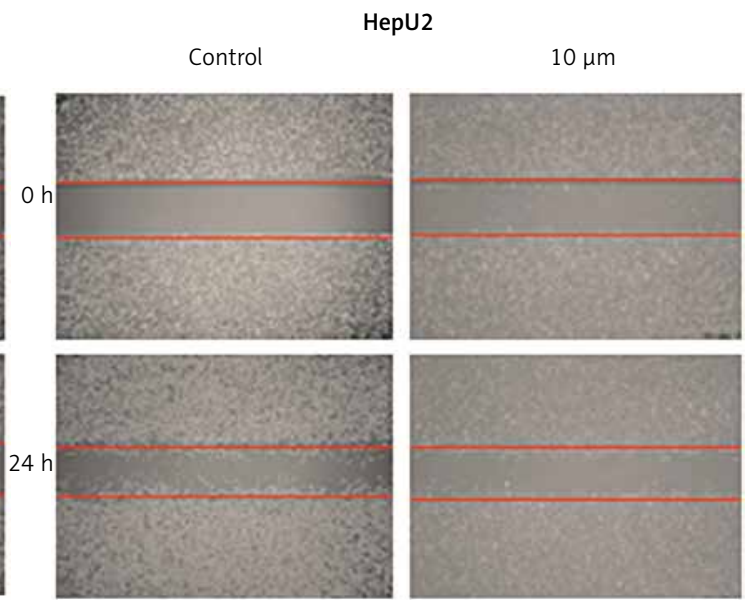

HepU2

Control

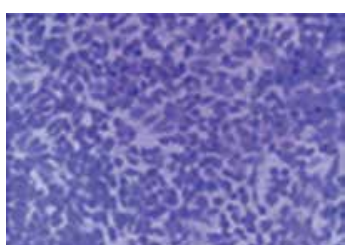

$10 \mu \mathrm{m}$

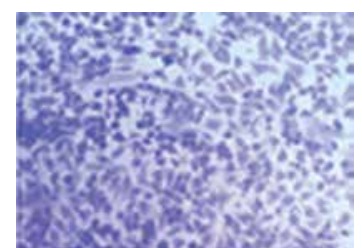

Figure 5. A - Images showing cell migration in HEPU1 and HEPU2 cells after sulforaphane exposure. B - Images showing cell invasion in HEPU1 and HEPU2 cells after sulforaphane exposure. Experiments were repeated three times
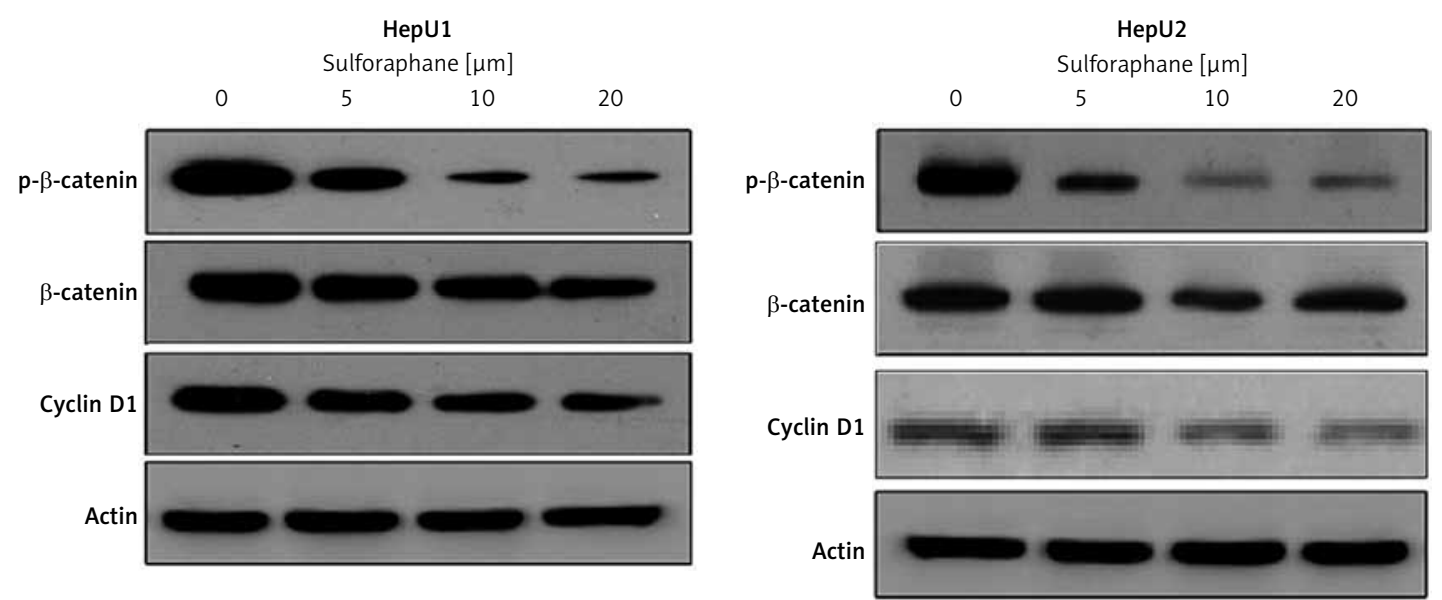

Figure 6. Western blotting assay indicating activity of $\beta$-catenin signaling pathway allied proteins after exposure to indicated sulforaphane doses. Experiments were repeated three times 
death. The $\beta$-catenin signaling pathway in HepU1 and HepU2 cells was monitored by western blotting assay. The results indicated that the protein expression of $\beta$-catenin, $p$ - $\beta$-catenin and cyclin D1 decreased sulforaphane dose dependently (Figure 6). Therefore, the results suggested that the sulforaphane blocked the $\beta$-catenin signaling pathway in both HepU1 and HepU2 cell lines.

\section{Discussion}

$\mathrm{HB}$ is a lethal liver malignancy occurring in children. The disease has been reported with higher incidence in the past decade, globally. Lack of treatment strategies and potential chemo-preventive agents necessitates the development of efficient chemotherapeutic agents. Eukaryotes possess only one known machinery to disintegrate the large intracellular protein aggregates and organelles that cannot be disposed of by the proteasome [18-20]. This machinery is termed "autophagy" and operates through lysosomal degradation. Hence, it is not surprising that microorganisms such as bacteria, protozoa and viruses that infect eukaryotes are degraded by autophagy [21, 22]. Autophagy allied proteins also provide miscellaneous features of immunity in multicellular organisms. Several proteins that regulate autophagy have been identified (around 32) in yeast. In autophagy and phagophore formation, the role of class III PI-3 kinases (notably Vps34 and its binding partner Atg6/Beclin-1) is comparatively well understood [23]. Vps34 plays an important role in membrane sorting in a cell but when Vps34 is complexed with Beclin-1 it exhibits active participation in autophagy. Formation of autophagosomes and upregulation of autophagy allied proteins such as Atgs, Beclin-1 and LC3 proteins hallmark the autophagic cell death [24, 25]. Similarly, apoptosis is another form of cell regulatory mechanism used to discard old, damaged, and malfunctioning cells. Apoptosis is mostly characterized by nuclear condensation, DNA fragmentation, membrane blebbing and plasma membrane rupture [26-28]. It is allied with increased activity of Bax (pro-apoptotic protein) and reduced $\mathrm{Bcl}-2$ (anti-apoptotic) activity. During apoptosis the caspases are activated and initiate the intrinsic pathway of apoptosis. As the apoptosis-inducing potential of sulforaphane was already known, its applications were investigated on HB. Therefore, the current study was instigated to evaluate the anticancer cancer potential of sulforaphane in human HB. Cellular viability of cancerous HepU1 and HepU2 and normal THLE-2 cells was determined by MTT assay, indicating potential viability and colony inhibition in the case of cancerous cells in contrast to normal cells via induction of apoptosis and autophagy. These findings are in agreement with previous studies wherein sulforaphane has been found to inhibit growth of the HEp-2 human epithelial carcinoma cell line [13]. In yet another study, Gamet-Payrastre et al. reported that sulforaphane treatment induces apoptosis in human colon cancer cells, further confirming our results [15]. The $\beta$-catenin signaling pathway is an important pathway, and drugs are being developed to target this pathway for the treatment of different cancers. Herein we found that sulforaphane blocks this pathway in HB cells. In addition to cell migration, cell invasion was also impaired by sulforaphane application. These findings are consistent with a previously carried out study wherein sulforaphane was shown to inhibit glioblastoma cell invasion [29].

In conclusion, the data obtained in the present study indicated that sulforaphane exerts anticancer effects on human HB cells. The anticancer effects were found to be mediated via induction of apoptosis, autophagy and inhibition of the $\beta$-catenin signaling pathway. Therefore, sulforaphane is a promising molecule in in vitro studies, which makes this substance a good candidate for further research in hepatoblastoma management.

\section{Acknowledgments}

Junshan Lin and Yali Xu-equal contribution.

\section{Conflict of interest}

The authors declare no conflict of interest.

\section{References}

1. Xie L, Onysko J, Morrison H. Childhood cancer incidence in Canada: demographic and geographic variation of temporal trends (1992-2010). Health Promot Chronic Dis Prev Can 2018; 38: 79-115.

2. Linabery AM, Ross JA. Trends in childhood cancer incidence in the U.S. (1992-2004). Cancer 2008; 112: 416-32.

3. Allan BJ, Parikh PP, Diaz S, Perez EA, Neville HL, Sola JE. Predictors of survival and incidence of hepatoblastoma in the paediatric population. HPB (Oxford) 2013; 15: 741-6.

4. Hung GY, Lin LY, Yu TY, Lee CY, Yen HJ, Horng JL. Hepatoblastoma incidence in Taiwan: a population-based study. J Chin Med Assoc 2018; 81: 541-7.

5. Spector LG, Birch J. The epidemiology of hepatoblastoma. Pediatr Blood Cancer 2012; 59: 776-9.

6. Hermann RE, Lonsdale D. Chemotherapy, radiotherapy, and hepatic lobectomy for hepatoblastoma in an infant: report of a survival. Surgery 1970; 68: 383-8.

7. Exelby PR, Filler RM, Grosfeld JL. Liver tumors in children in the particular reference to Hepatoblastoma and hepatocellular carcinoma: American Academy of Pediatrics Surgical Section Survey--1974. J Pediatr Surg 1975; 10: 329-37.

8. Qin WS, Deng YH, Cui FC. Sulforaphane protects against acrolein-induced oxidative stress and inflammatory responses: modulation of Nrf-2 and COX-2 expression. Arch Med Sci 2016; 12: 871-80. 
9. Conaway C, Yang YM, Chung FL. Isothiocyanates as cancer chemopreventive agents: their biological activities and metabolism in rodents and humans. Curr Drug Metab 2002; 3: 233-55.

10. Moy KA, Yuan JM, Chung FL, et al. Isothiocyanates, glutathione S-transferase M1 and T1 polymorphisms and gastric cancer risk: a prospective study of men in Shanghai, China. Int J Cancer 2009; 125: 2652-9.

11. Byun S, Shin SH, Park J, et al. Sulforaphene suppresses growth of colon cancer-derived tumors via induction of glutathione depletion and microtubule depolymerization. Mol Nutr Food Res 2016; 60: 1068-78.

12. Mondal A, Biswas R, Rhee YH, Kim J, Ahn JC. Sulforaphene promotes Bax/Bcl2, MAPK-dependent human gastric cancer AGS cells apoptosis and inhibits migration via EGFR, p-ERK1/2 down-regulation. Gen Physiol Biophy 2016; 35: 25-34.

13. Devi JR, Thangam EB. Mechanisms of anticancer activity of sulforaphane from Brassica oleracea in HEp-2 human epithelial carcinoma cell line. Asian Pac J Cancer Prev 2012; 13: 2095-100.

14. Qazi A, Pal J, Maitah MI, et al. Anticancer activity of a broccoli derivative, sulforaphane, in Barrett adenocarcinoma: potential use in chemoprevention and as adjuvant in chemotherapy. Transl Oncol 2010; 3: 389-99.

15. Gamet-Payrastre L, Li P, Lumeau S, et al. Sulforaphane, a naturally occurring isothiocyanate, induces cell cycle arrest and apoptosis in HT29 human colon cancer cells. Cancer Res 2000; 60: 1426-33.

16. Pledgie-Tracy A, Sobolewski MD, Davidson NE. Sulforaphane induces cell type-specific apoptosis in human breast cancer cell lines. Mol Cancer Therap 2007; 6: 1013-21.

17. Chiao JW, Chung FL, Kancherla R, Ahmed T, Mittelman A, Conaway CC. Sulforaphane and its metabolite mediate growth arrest and apoptosis in human prostate cancer cells. Int J Oncol 2002; 20: 631-6.

18. Milczarek M, Wiktorska K, Mielczarek L, et al. Autophagic cell death and premature senescence: new mechanism of 5-fluorouracil and sulforaphane synergistic anticancer effect in MDA-MB-231 triple negative breast cancer cell line. Food Chem Toxicol 2018; 111: 1-8.

19. Tanaka Y, Guhde G, Suter A, et al. Accumulation of autophagic vacuolesand cardiomyopathy in LAMP-2-deficient mice. Nature 2000; 406: 902-6.

20. Kuma A, Hatano M, Matsui M, et al. The role of autophagy during the early neonatal starvation period. Nature 2004; 432: 1032-6.

21. Deretic V, Levine B. Autophagy, immunity, and microbial adaptations. Cell Host Microbe 2009; 5: 527-49.

22. Virgin HW, Levine B. Autophagy genes in immunity. Nature Immunol 2009; 10: 461-70.

23. Backer JM. The reguation and function of class III PI3Ks: novelroles for Vps34. Biochem J 2008; 410: 1-17.

24. Radoshevich L, Murrow L, Chen N, et al. ATG12 conjugation to ATG3 regulates mitochondrial homeostasis and cell death. Cell 2010; 142: 590-600.

25. Fujita N, Itoh T, Omori H, Fukuda M, Noda T, Yoshimori T. The Atg16L complex specifies the site of LC3 lipidation for membrane biogenesisin autophagy. Mol Biol Cell 2008; 19: 2092-100.

26. Engel T, Henshall DC. Apoptosis, Bcl-2 family proteins and caspases: the ABCs of seizure-damage and epileptogenesis? Int J Physiol Pathophysiol Pharmacol 2009; 1: 97-115.

27. Liu JJ, Lin M, Yu JY, Liu B, Bao JK. Targeting apoptoticand autophagic pathways for cancer therapeutics. Cancer Lett 2011; 300: 105-14.
28. Wen X, Lin ZQ, Liu B, Wei YQ. Caspase-mediated programmedcell death pathways as potential therapeutic targets incancer. Cell Prolif 2012; 45: 217-24.

29. Li C, Zhou Y, Peng X, et al. Sulforaphane inhibits invasion via activating ERK1/2 signaling in human glioblastoma U87MG and U373MG cells. PLoS One 2014; 9: e90520. 